\title{
Exciton-Exciton Annihilation as a Probe of Exciton Diffusion in Large Porphyrin Nanorings
}

Giovanni Bressan, ${ }^{1}$ Michael Jirasek, ${ }^{2}$ Harry L. Anderson, ${ }^{2}$ Ismael A. Heisler ${ }^{3,}$ and Stephen R. Meech $^{1, *}$

${ }^{1}$ School of Chemistry, Norwich Research Park, University of East Anglia, Norwich NR4 7TJ, United Kingdom

${ }^{2}$ Department of Chemistry, University of Oxford, Chemistry Research Laboratory, Oxford OX1 3TA, United Kingdom ${ }^{3}$

${ }^{3}$ Departamento de Física, Universidade Federal do Paraná, Caixa Postal 19044, 81531-990 Curitiba, Parana, Brazil

Corresponding authors: s.meech@uea.ac.uk; heisler@fisica.ufpr.br

\begin{abstract}
The photophysical behavior of natural and artificial cyclic supramolecular structures has been intensively investigated in the past decade. Among the artificial structures, large fully $\pi$ conjugated porphyrin nanorings are of particular interest because of their electronic, structural and topological features, which make them suitable candidates for light harvesting applications. A number of factors affect the efficiency with which such structures harvest and transmit energy. For instance, under intense irradiation conditions, the efficiency of light harvesting can be quenched by competing processes, such as exciton-exciton annihilation.

Here we investigate the pump-fluence dependence on the transient absorption spectra of a series or porphyrin nanorings ranging in circumference between 13 and $52 \mathrm{~nm}$ (10-40 porphyrin units). This allowed the isolation of a fluence-dependent fast-decaying component in all but the smallest nanorings studied. This effect has been assigned to exciton-exciton annihilation and fit to a one-dimensional exciton diffusion model which confirms that the exciton size and/or its mobility are inversely proportional to the nanoring size.
\end{abstract}




\section{Introduction}

Cyclic supramolecular $\pi$-structures are nanosystems with unique topology $y^{1,2}$ and characteristics. ${ }^{3-5}$ The conformation of highly symmetric $\pi$-conjugated nanorings, sometimes displaying diameter-dependent strain, ${ }^{6}$ has a major impact on their optical and electronic properties, ${ }^{7,8}$ inducing new phenomena, absent in their linear counterparts, ${ }^{9}$ such as intensity borrowing due to coupling between their vibrational and electronic wavefunctions and reduced fluorescence quantum yields. ${ }^{10}$

Cyclic structures are common in nature as, for instance, in light harvesting complexes within the photosynthetic apparatus. The chromophores in these naturally occurring cyclic structures (mostly chlorophylls) are excitonically coupled, resulting in delocalization of their excited states, or excitons, over several chromophoric units. ${ }^{11-13}$ Such delocalized excitations facilitate energy transfer among the molecules that make up the photosynthetic apparatus. ${ }^{14-}$ 17 Bio-inspired synthetic materials mimicking naturally-occurring porphyrin-like cyclic nanostructures also display excitations with non-local character, ${ }^{9,10}$ and are thus good candidates for light harvesting and energy-transfer applications.

The performance of devices based on biomimetic nanorings will ultimately be connected to their properties, such as exciton size (delocalization) and charge mobility. These are, inturn, affected by the structure and the disorder experienced by the exciton on the nanoring. It has been reported that, when the number of repeating units in a fully conjugated porphyrin nanoring is greater than 24, the excitons can only delocalize across a section of the macrocycle, resembling the behavior of an exciton moving on an infinite linear chain. ${ }^{6}$ Moreover, large porphyrin nanorings, while thought of as having circular shape (and symmetry), in fact display a range of folding dynamics, and can form nested and stacked structures. $^{18}$

Exciton-Exciton Annihilation (EEA) is a photophysical phenomenon which can be detrimental to the light harvesting efficiency of extended $\pi$-conjugated structures, ${ }^{19,20}$ and which has been shown to have a significant effect on the photodynamics of linear extended chromophore chains. ${ }^{21,22}$ EEA takes place when two excitons, due to their spatial proximity, combine their energies allowing access to higher electronic excited states, which subsequently decay back to their first excited state by fast internal conversion (IC). In order to conserve total energy, while one exciton is promoted to a higher excited state, the other one is down-converted to its electronic ground state; consequently EEA is an energy quenching mechanism. ${ }^{19,23}$ The EEA process is shown schematically in Figure 1. 


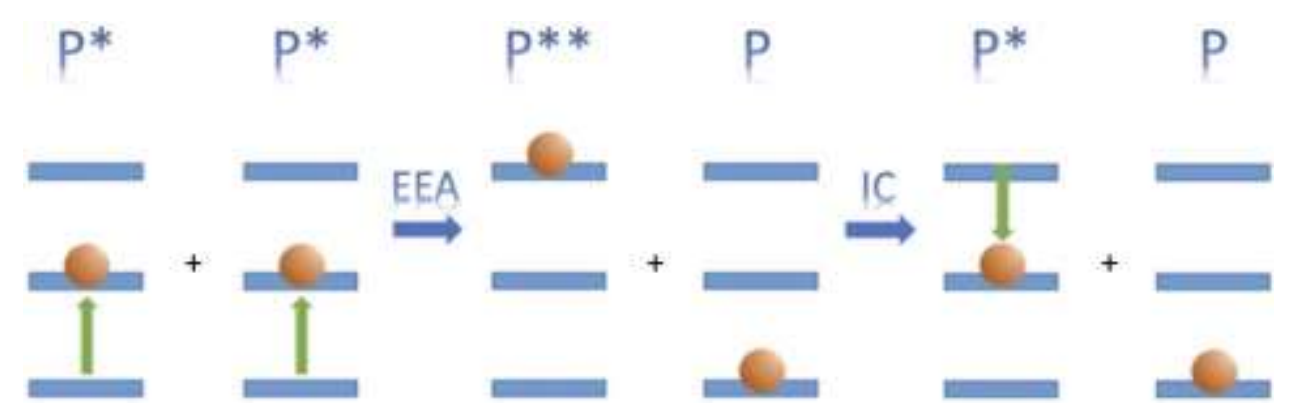

Figure 1 EEA relaxation dynamics after light-induced excitation (upwards vertical arrows). Two $S_{1}$ excitons $\left(P^{*} P^{*}\right)$ interact such that one is promoted to its second excited state, whereas the other one is de-excited to the ground state ( $\left.P^{* *} P\right)$. In a second step, internal conversion takes place (downwards vertical arrow). This pathway ends up in the final configuration $\left(P^{*} P\right)$, where one quantum of excitation energy is missing; EEA is thus a quenching mechanism.

The EEA dynamics, assuming that the concentration is low enough to neglect interactions between different nanorings, have a dependence on both exciton collision rate and exciton mobility (within a nanoring). ${ }^{20}$ Both parameters are influenced by size and topology of the nanoring. EEA has been predicted and experimentally observed in a range of excitonic supramolecular systems of different nature and dimensionality, ${ }^{22-26}$ and has been modeled considering either the exciton lifetime or the exciton diffusion time as the rate-determining step. ${ }^{19,20}$

In this paper we present results of femtosecond transient absorption (fsTA) spectroscopy, performed as a function of increasing pump fluence, on a series of fully conjugated porphyrin nanorings, ranging in size from 10 to 40 repeating units. The EEA dynamics have been observed and characterized, allowing us to determine the threshold fluence at which EEA occurs in each nanoring. Further, the EEA dynamics have been analyzed assuming a onedimensional exciton diffusion model, modified to account for the nanoring circumference, allowing us to retrieve the exciton diffusion coefficient on each nanoring. The results broadly agree with literature data, obtained through fluorescence upconversion measurements on the same series of nanostructures. ${ }^{6}$ We confirm that the larger structures have excitations partially localized on a segment of the nanoring, spanning ca. 20 chromophores; the excitation on the smaller, 10-membered, nanoring is fully delocalized, consistent with earlier steady state data. ${ }^{6}$ Moreover, the exciton diffusion coefficient is found to be inversely proportional to the nanoring size, confirming the hypothesis that conformational disorder can reduce exciton mobility and/or size, due to the creation of trap sites which cause the excitons to localize on a segment of the macrocycles.

\section{Experimental methods}

All time-resolved measurements were performed on a visible fsTA experimental setup based on the design by Riedle. ${ }^{27}$ A chirped pulse amplifier (Spitfire ACE, Spectra-Physics) seeded by a Ti:sapphire oscillator (MaiTai, Spectra-Physics) and pumped by a Q-switched doubled Nd:YLF laser (Empower, Spectra-Physics) generates $120 \mathrm{fs}$ pulses centered at $800 \mathrm{~nm}$ 
at a repetition rate of $1 \mathrm{kHz}$. This drives a commercial nonlinear optical parametric amplifier (NOPA, TOPAS White, Light Conversion) and a commercial collinear optical parametric amplifier (OPA, TOPAS Prime, Light Conversion). The NOPA pulses, used as the pump (shown as solid magenta line in Figure 2), are $20 \mathrm{fs}$ long and centered at $700 \mathrm{~nm}$ while the OPA pulses, used as probe, are $75 \mathrm{fs}$ long and centered at $1200 \mathrm{~nm}$. The NOPA pulses were recompressed close to the Fourier transform limit at the sample position using a folded prism compressor, and characterized via transient grating frequency resolved optical gating (TG-FROG) measurements in a $1 \mathrm{~mm}$ optical path fused silica cell filled with cyclohexane as the nonlinear medium. The broadband probe pulses are obtained by focusing the OPA pulses at $1200 \mathrm{~nm}$ into a $3 \mathrm{~mm}$ thick static sapphire plate to generate a white light continuum spanning more than an octave between 550 and $1200 \mathrm{~nm}$.

Reflective optics were used to steer pump and probe pulses to the interaction region at the sample position, thus minimizing dispersion. Pump and probe pulses were focused to overlapping spots with FWHM of $105 \pm 8$ and $50 \pm 2 \mu \mathrm{m}$, respectively. The relative pumpprobe polarization angle was set to $45^{\circ}$ with a $\lambda / 2$ zero order waveplate. After the sample, the two polarization components of the probe were split by a polarizing cubic beam-splitter, and each polarization is simultaneously dispersed in a prism and measured at $1 \mathrm{kHz}$ by two back-thinned full-frame transfer CCD cameras (Stresing). An optical chopper set at $500 \mathrm{~Hz}$ was used to modulate the pump beam, allowing the calculation of transient absorption spectra between sequential pairs of probe pulses. The isotropic signal was then calculated as $S_{\text {iso }}=$ $\frac{1}{3}\left(S_{\|}+2 S_{\perp}\right)$.

The porphyrin nanorings were synthesized as previously reported ${ }^{18,28,29}$ and dissolved in toluene with $1 \%$ by volume of pyridine to coordinate to the $\mathrm{Zn}$, atoms and thus prevent aggregation. The solutions obtained were placed in $1 \mathrm{~mm}$ fused silica static cells for both timeresolved and steady-state measurements, adjusting their concentration to obtain a peak OD around 0.3. The steady-state measurements were performed using a commercial UV-visible spectrometer (Lambda XLS, Perkin-Elmer).

\section{Results and Discussion}

\section{Steady-state absorption}

A general scheme of the molecular structure of the nanorings is shown in Figure 2a. Normalized steady-state absorption spectra of porphyrin nanorings with 10, 20, 30 and 40 porphyrin units are shown in Figure $2 b$. The normalized spectrum of the pump pulse used for the fsTA measurements is also shown in $2 b$. 
(a)
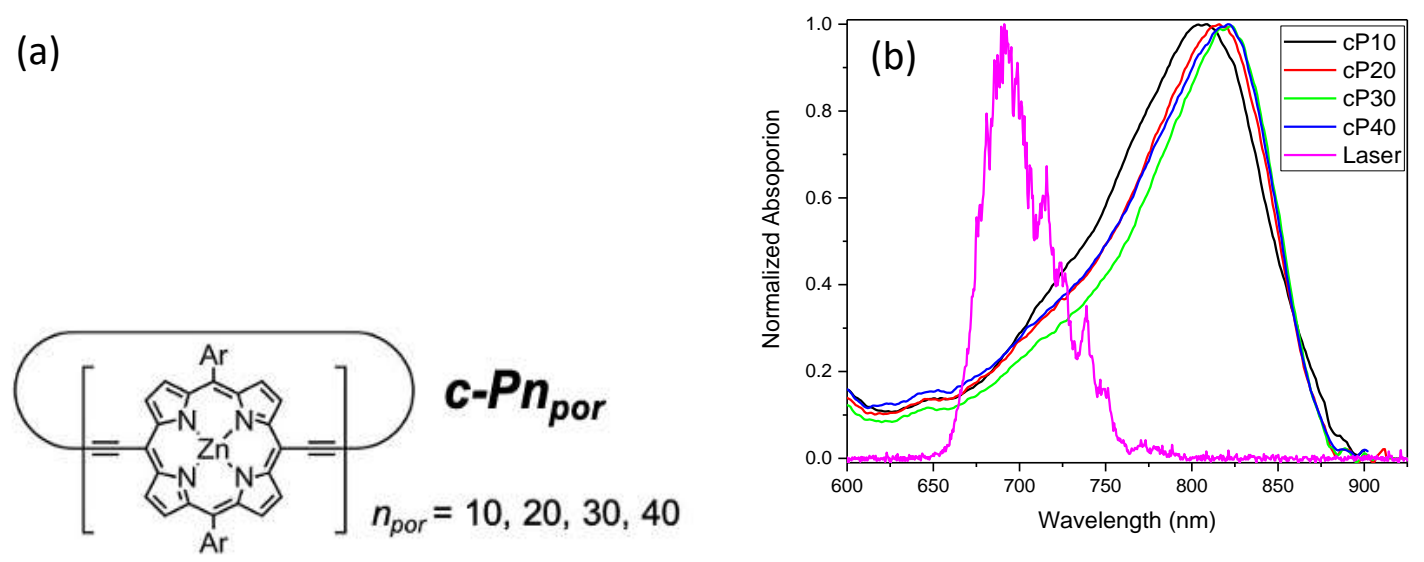

Figure 2 (a) Chemical structure of the cyclic conjugated porphyrin nanorings. Ar =3,5-bis(octyloxy)phenyl. (b) Normalized steady-state absorption spectra of c-P10 (solid red line), c-P20 (solid orange line), c-P30 (solid green line) and c-P40 (solid blue line). The spectra were measured at room temperature in toluene with $1 \%$ volume pyridine. The normalized spectrum of the NOPA used as pump for the fsTA measurements is shown in magenta as comparison.

Due to the circular symmetry of the nanorings, the excited state energy-levels can be conveniently described by the Hückel molecular orbital theory for $\pi$-conjugated molecules. ${ }^{10,30}$ This theory indicates that only transition dipole moments to the two degenerate $\left(S_{2}\right)$ nanoring molecular orbitals, with two nodes $(k= \pm 1)$, of orthogonal polarization ( $x$ and $y$ transitions) carry significant oscillator strength whereas transitions to the lowest unoccupied molecular orbital (LUMO, $S_{1}$ ), which has no nodes $(k=0)$ has a transition dipole moment strength equal to zero. Furthermore, the energy gap between ground and excited states decreases a function of $1 / n$, where $n$ is the number of coupled chromophores over which the excitation can delocalize. ${ }^{6}$ Therefore, the red-shift observed in the steady-state absorption spectra (Figure $2 b$ ) for increasing nanoring size indicates the increase in excitation delocalization. However, this effect saturates and is disrupted due to structural inhomogeneity around the nanoring with 30 chromophore units, as no further redshift was observed when going from c-P30 to c-P40.

The bandwidth of the steady-state electronic spectra of the nanorings reflects both exchange narrowing ${ }^{31}$ phenomena, $\left(\propto \sqrt{1 / n_{\text {por }}}\right)$ observed as the nanoring size is increased, and broadening due to the larger amount of static disorder experienced by larger rings. The net result of this is a progressive narrowing going from c-P10 to c-P30 followed by the broadening observed in C-P40.

In previous work on the same series of macrocycles, their steady-state spectra were employed to compute a Meier plot, which points to an effective conjugation length of ca. 20 porphyrin repeating units. ${ }^{6}$

\section{Transient Absorption}


The transient absorption spectra of $\mathbf{c - P 1 0 , - 2 0 , - 3 0}$ and $-\mathbf{4 0}$, at various delay times $(T)$ between 0.1 and 500 ps, are shown in Figure $3 a, b, c$ and d, respectively. These measurements were obtained for a low pump energy around $15 \mathrm{~nJ}$, in order to avoid EEA signals, as will be discussed further below.

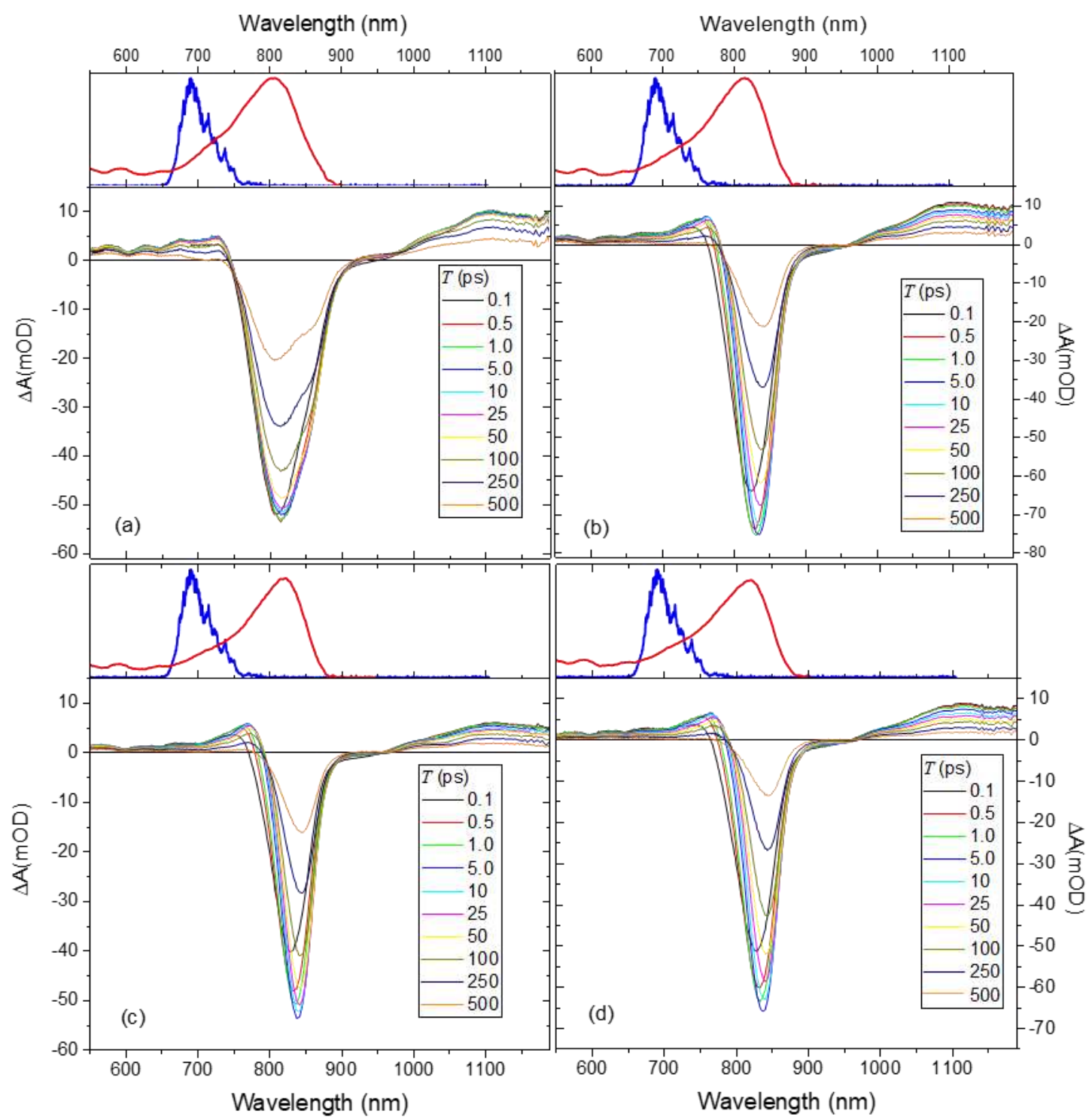

Figure 3 fsTA spectra after excitation with pump pulses shown in magenta in Figure 2, at ten increasing pump-probe delay times (T) between 0.1 and 500 ps of c-P10 (a), c-P20 (b), c-P30 (c) and c-P40 (d) are shown.

The main feature in all transient absorption spectra is a prominent negative band, present from the earliest times (as shown for $T=0.1 \mathrm{ps}$ ) and peaking at $811 \mathrm{~nm}$ for $\mathbf{c - P 1 0}$, at $825 \mathrm{~nm}$ for $\mathbf{c - P 2 0}$, at $830 \mathrm{~nm}$ for $\mathbf{c - P 3 0}$ and $\boldsymbol{c - P 4 0}$. These signals are spectrally matching the maxima of the steady-state absorption spectra of each nanoring, and are thus assigned to a bleaching of the electronic ground states (GSB). However, the negative signals extend further towards the red than the steady-state absorption, in a region where we expect to find fluorescence emission from the nanorings (see SI of Parkinson et $a l .{ }^{6}$ ), and so is assigned to a stimulated emission (SE) contribution. Furthermore, a fluorescence quantum yield increase was 
observed when the nanoring size increases. ${ }^{6}$ One can also observe that the bandwidth of the negative GSB + SE signal is quite broad for c-P10 and narrows for c-P20-c-P40. Bandwidth narrowing and fluorescence quantum yield enhancement are characteristic features manifested in J-coupled aggregates. ${ }^{32}$ Broad positive signals extending to the red from about $950 \mathrm{~nm}$ and to the blue from about $750 \mathrm{~nm}$ correspond to excited state absorption (ESA).

In order to determine the time constants associated with dynamical evolution of the transient absorption spectra, the spectra were fit globally using the software Glotaran. ${ }^{33}$

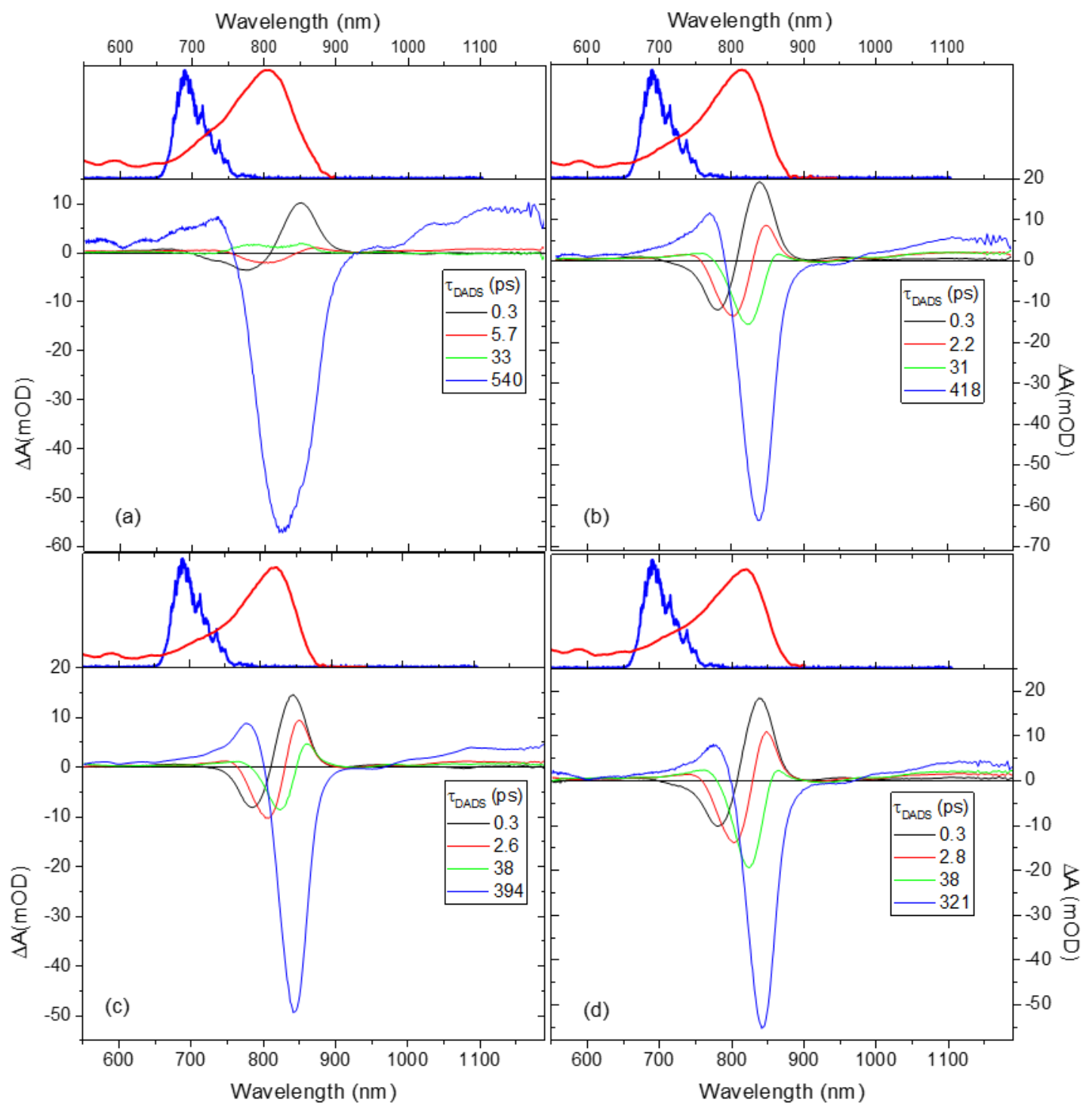

Figure 4 Evolution associated difference spectra obtained by global fitting of the fsTA data shown in Figure 3. (a) is c-P10, (b) is C-P2O, (c) is C-P30 and (d) is C-P4O.

Figure 4 presents the retrieved decay associated difference spectra (DADS) that best describe the dynamical evolution of the transient absorption spectra (the evolution associated decay spectra, EADS are shown in the SI, Figure S1). The spectral evolution occurs on multiple timescales. For all nanorings, the longest component (hundreds of picoseconds) corresponds 
to the excited state population relaxation back to the ground state, as this timescale accounts for the GSB recovery and SE + ESA relaxation.

In Figure 4, the two shortest components (black curve with $\tau_{\text {DADS }} \sim 0.3 p s$ and red curve with $\tau_{\text {DADS }} \sim 3.0 \mathrm{ps}$ ) have a shape that is negative around $771 \mathrm{~nm}$ and positive around $840 \mathrm{~nm}$. Such a DADS shape indicates that the transient absorption spectra undergo an amplitude increase (for both positive and negative bands) and some spectral redshift in the region from 700 to $900 \mathrm{~nm}$. This can clearly be seen in Figure 3, where during the first $\sim 3 \mathrm{ps,} \mathrm{the} \mathrm{positive}$ band at $771 \mathrm{~nm}$ and the negative band at $840 \mathrm{~nm}$ increase in amplitude. Therefore, these two DADS components account for energy redistribution in the singlet excited state manifold. A number of physical processes may contribute, which are difficult to distinguish and disentangle. Apart from intramolecular vibrational energy redistribution and vibrational cooling, electronic energy transfer within the excited state manifold might be happening. Some of those states could have a stronger transition dipole moment than the state initially excited. Such a relaxation will translate to an increased amplitude of transition dipole moment leading, therefore, to the SE and ESA amplitude increase observed.

A third component, with a time constant around $\sim 35 \mathrm{ps}$, contributes weakly to $c-P 10$, and is completely absent for $\mathbf{C}-\mathbf{P 6},{ }^{34}$ but becomes quite prominent as the nanoring size increases. We speculate that this $\sim 35$ ps component accounts for the exciton motion across the nanoring towards lower energy sites. For the bigger nanorings (c-P20-c-P40), the exciton might not be fully delocalized across the nanoring due to, for instance, increased structural disorder, therefore the exciton can diffuse around the nanoring; ${ }^{35}$ we return to this point below when considering EEA.

Finally, the broad and featureless positive ESA signals extending from the higher wavelength side of the transient spectra towards the near infrared (NIR), have broad maxima at ca. $1100 \mathrm{~nm}$ for all the nanorings. Decay of this ESA reflects excited state population relaxation followed by inter system crossing (ISC) to populate a triplet state, occurring on the hundreds of picoseconds timescale; a two-state (singlet to triplet) relaxation is supported by observations of isosbestic points at ca. $960 \mathrm{~nm}$ (Figure 3). Experimental evidence of formation of triplet states via inter system crossing, happening on comparable timescales, has been observed in both $\mathrm{Zn}$ porphyrin monomers ${ }^{36}$ and six-membered porphyrin nanorings. ${ }^{34,37}$ The final DADS component, corresponding to tens of nanoseconds, is summarized for all the nanorings in Figure S2. The incomplete recovery of the GSB at the longest measured pumpprobe delay times, while the ESA is substantially quenched after 250 ps (see Figure 3 and Figure S2), is consistent with this hypothesis of population being transferred to a long-lived triplet state. 
The NIR region of the fsTA spectra are due solely to singlet excited state absorption during the first 200 ps. For this reason we chose to investigate pump fluence-dependent dynamics focusing on the behavior of the $1100-1200 \mathrm{~nm}$ region of the ESA.

\section{Intensity Dependence: Exciton diffusion and annihilation}

Increasing the pump fluence leads to an increased probability that more than one exciton is created on a single nanoring. The average number of excitons per nanoring $\left\langle n^{*}\right\rangle$ generated by the pump pulse of known energy, ${ }^{20}$ is required to model the observed EEA dynamics (below); it can be calculated from the steady-state absorption spectrum of each nanoring using Equation 1:20

$\left\langle n^{*}\right\rangle_{\text {vol }}=\frac{N_{\text {pump }}}{\pi d r^{2} \text { probe }}\left[1-10^{-O D}\left(\lambda_{\text {pump }}\right)\right]$

where $N_{\text {pump }}$ is the number of photons in the sample region in which pump and probe pulses are overlapping, $d$ is the sample thickness $(1 \mathrm{~mm})$ and $O D\left(\lambda_{\text {pump }}\right)$ is the sample optical density at the central wavelength $\left(\lambda_{\text {pump }}=720 \mathrm{~nm}\right)$ of the NOPA pulses used, ca. 0.1 (Figure 2).

$N_{\text {pump }}$ is defined as:

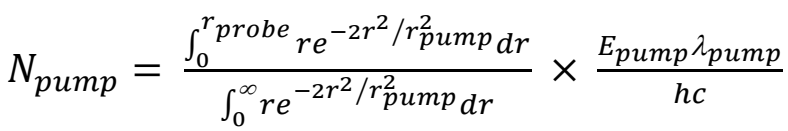

where the $r$ are pump and probe radii (full width half maxima of $r_{\text {pump }}=105 \mu \mathrm{m}$ and of $r_{\text {probe }}$ $=50 \mu \mathrm{m})$ and $E_{\text {pump }}$ and $\lambda_{\text {pump }}$ are defined as the optical energy and the central wavelength of the pump (NOPA) pulse. From Equation 1 the value of $\left\langle n^{*}\right\rangle_{v o l}$ is linearly dependent on the optical energy of the pump pulse $E_{\text {pump }}$, from which, using Equation 3, it is possible to retrieve the average number of excitons per ring in the nanorings.

$\left\langle n^{*}\right\rangle_{\text {mol }}=\frac{\left\langle n^{*}\right\rangle_{\text {vol }}}{c_{\text {nanoring }}}$

in which $c_{\text {nanoring }}$ is the molar concentration of each nanoring sample, which is obtained knowing the molar absorption coefficient $\varepsilon_{\text {nanoring }}(\lambda)$, using the Beer-Lambert relation. The values for $\varepsilon_{\text {nanoring }}(\lambda)$ and $\left\langle n^{*}\right\rangle_{\text {mol }}$ for each value of optical energy $E_{\text {pump }}$ used for c-P10, 20, 30 and 40 are given in Table 1.

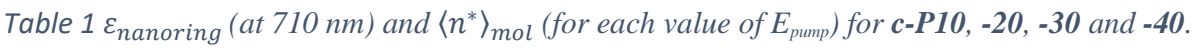

\begin{tabular}{ccc}
\hline$\varepsilon_{\text {nanoring }}\left[M^{-1} \boldsymbol{c m}^{-1}\right]$ & $E_{\text {pump }}[\boldsymbol{n J}]$ & $\left\langle\boldsymbol{n}^{*}\right\rangle_{\text {mol }}$ \\
\hline 10 & 0.02
\end{tabular}




\begin{tabular}{|c|c|c|c|}
\hline \multirow[t]{3}{*}{$c-\mathrm{P} 10$} & \multirow[t]{3}{*}{630650} & 30 & 0.06 \\
\hline & & 50 & 0.10 \\
\hline & & 70 & 0.15 \\
\hline & \multirow{3}{*}{1261301} & 10 & 0.04 \\
\hline \multirow[t]{3}{*}{$c-P 20$} & & 50 & 0.22 \\
\hline & & 70 & 0.31 \\
\hline & \multirow{4}{*}{1891952} & 15 & 0.08 \\
\hline \multirow[t]{4}{*}{$c-\mathrm{P30}$} & & 30 & 0.17 \\
\hline & & 63 & 0.35 \\
\hline & & 74 & 0.46 \\
\hline & \multirow{4}{*}{2522602} & 10 & 0.07 \\
\hline \multirow[t]{3}{*}{$c-\mathrm{P} 40$} & & 30 & 0.21 \\
\hline & & 50 & 0.36 \\
\hline & & 70 & 0.50 \\
\hline
\end{tabular}

From these data, one can conclude that there is a significant probability that, for higher fluences and for larger nanorings, more than one exciton is generated on a single nanoring. This then increases the probability of EEA, whose effect is to promote an additional excited state relaxation channel. This is indeed what happens experimentally; a faster decay component, observed as a steeper slope of the traces at early times, in the excited state absorption between 1100-1200 nm for c-P20-40 is observed, see Figure 5.
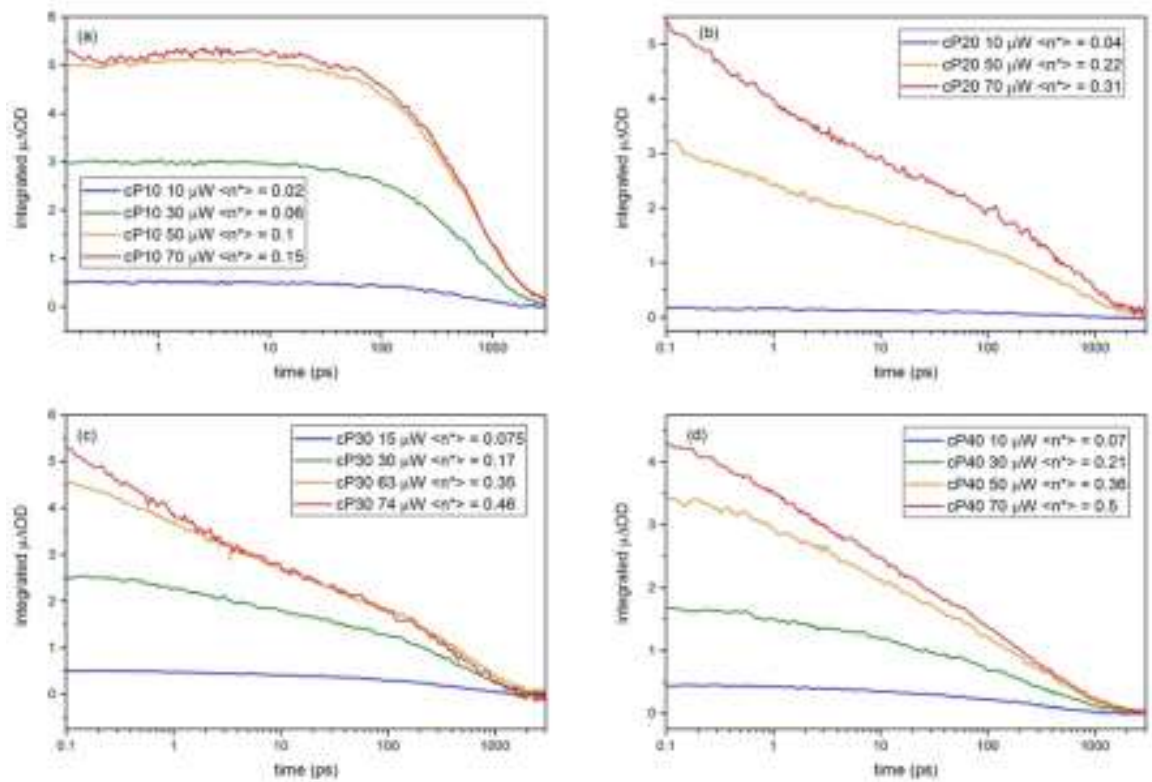

Figure 5: fsTA ESA integrated traces $(1100-1200 \mathrm{~nm})$ of c-P10 (a), c-P20 (b), c-P30 (c) and c-P40 (d) at increasing pump fluence. Time axis is shown on a log scale to emphasize the differences between the traces at early times. 
This effect does not occur in either $\mathbf{c}-\mathbf{P 6 ^ { 3 4 }}$ or $\mathbf{c - P 1 0}$. Also, no fast decaying component is observed in measurements at low fluences. Similar fluence-dependent fast dynamics have been observed in linear perylene bisimide and squaraine aggregates ${ }^{20,23}$ and in lightharvesting complexes, ${ }^{38,39}$ and have been interpreted and modeled as EEA.

As discussed above (see Figure 1), when EEA occurs, one exciton is promoted to higherlying electronic excited states which quickly decay back to $S_{1}$, while the other is de-excited to the ground state. The net effect is the loss of one half of the excitons involved in EEA dynamics. Engels and Wolter derived a rate equation for the number of excitons $n(T)$ as a function of time. ${ }^{22,25}$

$\frac{d n^{*}(T)}{d T}=-\frac{n^{*}(T)}{\tau}-\frac{1}{2} \gamma(T) n^{* 2}(T)$

in which $n^{*}(T)$ is the number of excitons as a function of the pump-probe delay time, $\tau$ is the lifetime of the $S_{1}$ state in the low-fluence limit, and $\gamma(T)$ is the annihilation rate, which can be time dependent. The factor of $1 / 2$ is introduced to account for the fact that every EEA event causes the loss of one of the two interacting excitons. Given that the nanoring can be thought as one-dimensional infinite linear chain, it is reasonable to assume a one-dimensional diffusive motion of the exciton along (one portion of) the chain, which allows us to use Equation 5 as the annihilation rate. ${ }^{22}$ Equation 5 has been derived from the equation of motion for a one-dimensional diffusion problem on a semi-infinite 1D slab with a perfect sink, using the appropriate boundary conditions. ${ }^{25}$

$\gamma(T)=\frac{1}{l N_{0}} \sqrt{\frac{8 D}{\pi T}}$

The annihilation rate depends on the diffusion coefficient, $D$, the nanoring circumference, $L$, which replaces the lattice parameter, $l$, in the original model, and the number of nanorings per unit volume, $N_{0}$. The nanoring circumference is included in our modified model because $N_{0}$ is the volume density of rings, rather than of porphyrin units. So, the distance between two neighboring porphyrins has to be multiplied by the number of porphyrins contained in each ring in order to make Equation 5 and the annihilation rates used by Wolter ${ }^{22}$ and Engel ${ }^{25}$ equivalent $\left(L / n_{\text {por }}=l\right.$ and $N_{0} \times n_{\text {por }}=N_{\text {por }}$, in which $n_{\text {por }}$ is the number of porphyrin units per ring, $N_{\text {por }}$ is the volume density of porphyrin units where $l$ is the lattice parameter of the original mode ${ }^{22,25}$ ). Equations 4 and 5 were developed to model the behavior of excitons placed on infinite linear chains, but with these adjustments they can be applied to model dynamics taking place in these nanorings, given the absence of end groups. Integration of Equation 4 and substitution of Equation 5 yields:

$n(T)=\frac{n_{0} e^{-T / \tau}}{1+\frac{n_{0}}{L N_{0}} \sqrt{2 D \tau} \operatorname{erf}\left(\sqrt{\frac{T}{\tau}}\right)}$ 
here, $n_{0}$ is the number density of excitons created by the pump pulse at $T=0$. The integrated optical density, proportional to the integrated fsTA signal, will be:

$I(T)=A \times \frac{e^{-T / \tau}}{1+\frac{\left\langle n^{*}\right\rangle_{\operatorname{mol}}}{L} \sqrt{2 D \tau} \operatorname{erf}\left(\sqrt{\frac{T}{\tau}}\right)}$

where $A$ is a rescaling factor to match the model and the experimental integrated traces at $T$ $=0.15 \mathrm{ps}$ (this $T$ is used to avoid effects due to cross-phase modulation closer to time zero), $\left\langle n^{*}\right\rangle_{m o l}$ is the average number of excitons per nanoring (see Table 1 ) and $\operatorname{erf}$ is the error function. The numerical behavior of such a one-dimensional exciton diffusion model as a function of diffusion coefficient, average exciton number per ring, ring size and $\mathrm{S}_{1}$ lifetime is illustrated in Figure S3.

The evolution of the integrated NIR ESA between 1100-1200 $\mathrm{nm}$ has been analyzed to infer pump fluence-dependent exciton decay dynamics. The signal has been integrated over a relatively broad spectral region $(1100-1200 \mathrm{~nm})$ rather than a single time trace at a given wavelength, allowing a focus on population rather than the aforementioned spectral shifts occurring at early delay times.

The one-dimensional diffusive model in Equation 7 does not account for a multiexponential relaxation of $S_{1}$, thus, in order to compare it to the experimental results, it is necessary to separate the decay of the first excited state from the EEA dynamics. To do so, measurements taken at different pump fluences (ranging from 10 to $70 \mathrm{~nJ} /$ pulse, note that 1 $\mu \mathrm{W}$ corresponds to $1 \mathrm{~nJ}$, given the laser repetition rate of $1 \mathrm{kHz}$ ) have been recorded for each sample, and the integrated trace obtained at the lowest fluence was then rescaled to give the best match with the long time behavior ( $T \geq 50 \mathrm{ps}$ ) for each integrated traces obtained at higher pump fluences. This 'tail matching' allowed subtraction of the two traces to isolate the multi-exciton dynamics to be modeled.

The lower pump fluence limit of $10 \mathrm{~nJ}$ was chosen as a compromise between obtaining an acceptable signal-to-noise ratio (SNR) without exceeding the values of $10 \%$ in $\left\langle n^{*}\right\rangle_{\text {mol }}$ (see Table 1). Tail-matched, integrated and rescaled traces for $\mathbf{c}-\mathbf{P 1 0}$, in which no pump fluencedependent dynamics have been observed, and for C-P40, showing clear early time pump fluence dependent behavior, are shown in Figure 6 (left and right columns, respectively). 

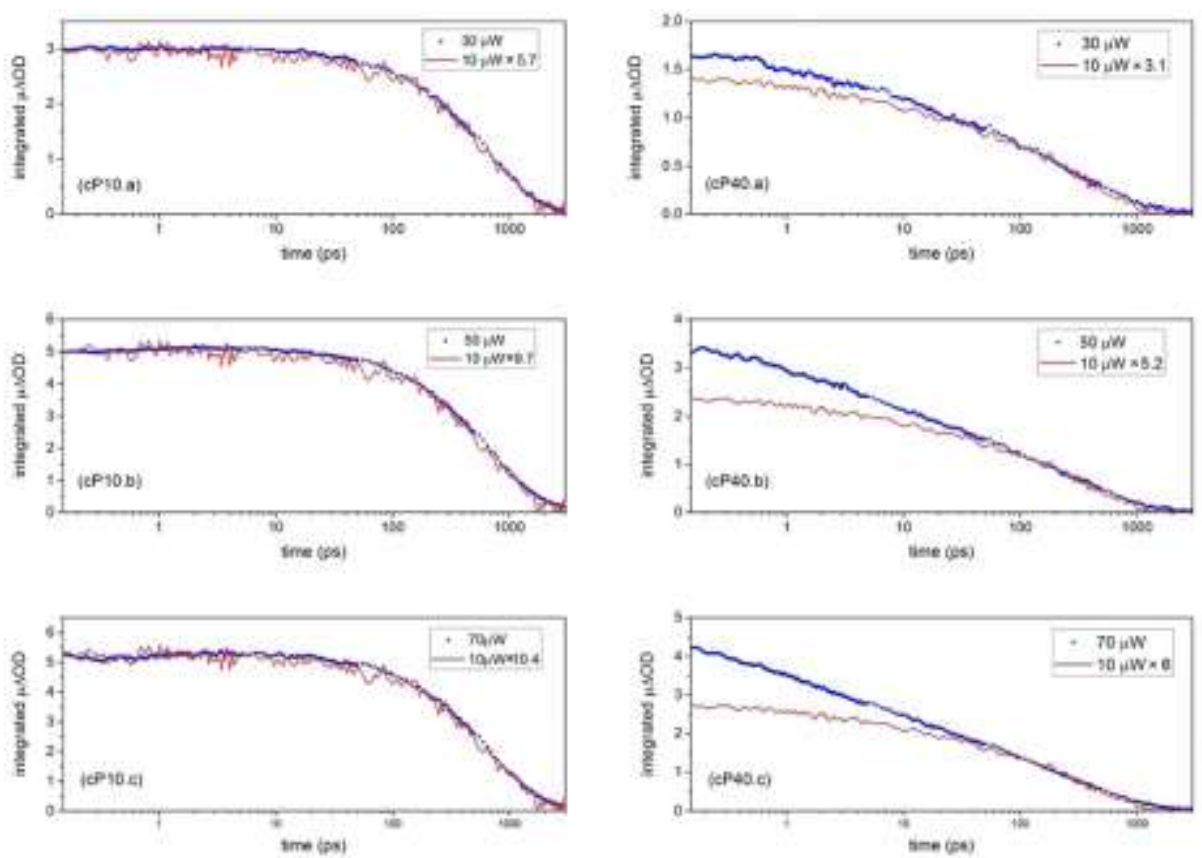

Figure 6 Comparison between rescaled $10 \mathrm{~nJ}$ (red solid line) and higher (blue dots) pump fluence fsTA traces (integrated 1100 $1200 \mathrm{~nm}$ ) for c-P10 (left) and c-P40 (right). The rescaling factors have been chosen in order to tail match the $10 \mathrm{~nJ}$ trace with the traces measured at higher pump powers. The abscissae are on a log scale to highlight early time dynamics. The fluence independence/dependence in the c-P10/c-P40 rings is obvious.

The tail matching procedure, and subsequent subtraction, have been applied to the dataset of $\boldsymbol{c}$-P10-c-P40 (see Figure S4 for all the rescaled integrated traces) and the resulting traces have been fit to the 1D diffusion model presented in Equation 7. The subtracted data and corresponding fitting curves (not for c-P10) are juxtaposed in Figure 7.

The first noticeable difference between c-P10 and c-P20-40 looking at Figure 7 is that the tail-matching and subtraction procedure allowed extraction of a fast decaying component

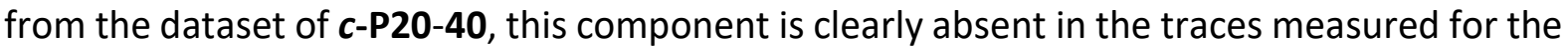
smallest nanoring studied, $\mathbf{c}-\mathbf{P} \mathbf{1 0}$. This can be readily explained if in $\mathbf{c - P 1 0}$ the exciton is delocalized over the whole nanoring structure; thus, even if two excitons were created on the same nanoring, they would instantaneously annihilate. In contrast, the fast decay due to EEA

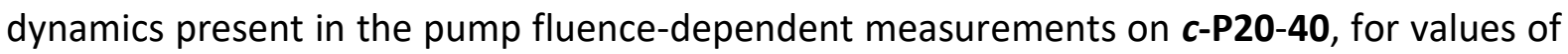
$T$ between 0.15 and $10 \mathrm{ps}$ are well fit by the one-dimensional diffusive exciton motion on a nanoring (Equation 7 and Figure 7).

To disentangle the single exciton population dynamics, whose lifetime is a variable of the fitting model, from the fast EEA process, the lifetime $\tau$ of the $S_{1}$ population in absence of EEA, has to be determined. The integrated ESA trace measured at the lowest fluence, at which EEA does not take place, are fit to a biexponential decay, followed by weighted averaging (using the amplitude of their pre-exponential factors as weighting parameters) of their $\tau_{1}$ and $\tau_{2}$. The initial average number of excitons per ring $\left\langle n^{*}\right\rangle_{m o l}$ has been calculated from steadystate data and pump-probe spots overlap integrals as previously described in Equations 2 and 
3 and tabulated in Table 1 . The nanoring circumference $L$ was obtained by multiplying the distance between two adjacent chromophores $(1.3 \mathrm{~nm})$ on a linear chain belonging to the same family of fully conjugated porphyrin oligomers. The only free parameter of the fit is then the exciton diffusion coefficient $D$. All the subtracted traces and their fits are shown in Figure 7 and all the parameters are summarized in Table 2.
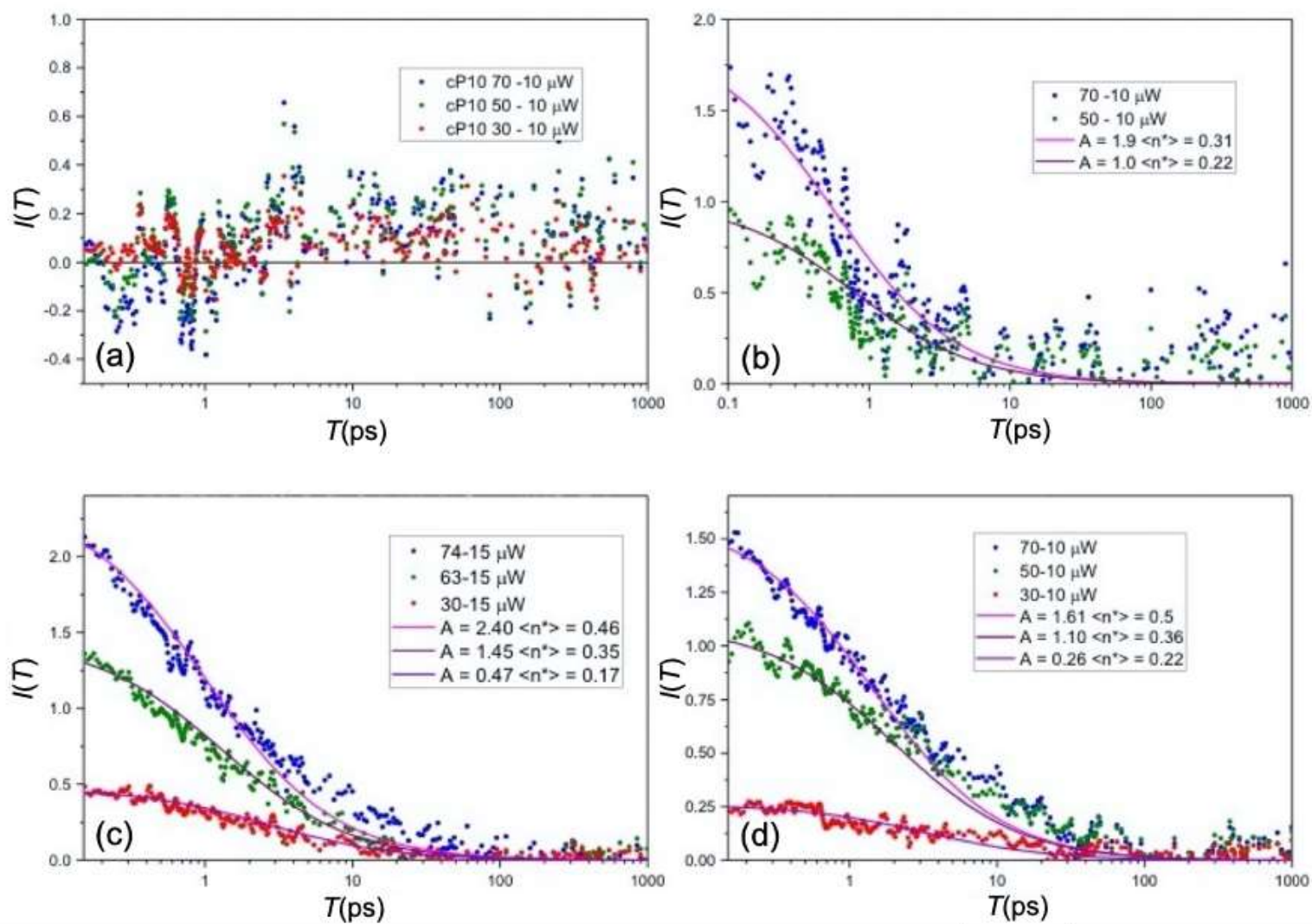

Figure 7 Subtracted integrated traces for c-P10 (a), and fits (using Equation 7) for c-P20 (b), c-P30 (c) and c-P40 (d) at different pump fluences. The parameters used for the fits are summarized in Table 2. The abscissae are on a log scale to highlight early time dynamics.

Table 2 Parameters for the 1D diffusion fit applied to the subtracted integrated NIR ESA data of c-P20, $-\mathbf{3 0}$ and $\mathbf{4 0}$. Fit results shown in Figure 7.

\begin{tabular}{lllllll}
\hline & $\begin{array}{l}\text { Fluence } \\
\text { subtraction (nJ) }\end{array}$ & $\left\langle n^{*}\right\rangle_{\text {mol }}$ & $A$ & $\tau[\mathrm{ps}]$ & $L[\mathrm{~cm}]$ & $D\left[\mathrm{~cm}^{2} / \mathbf{s}\right]$ \\
\hline c-P20 & $\mathbf{7 0 - 1 0}$ & 0.31 & 1.90 & 350 & $2.6 \times 10^{-6}$ & $3.00 \times 10^{-2}$ \\
& $\mathbf{5 0 - 1 0}$ & 0.22 & 1.01 & & & \\
\hline & $\mathbf{7 4 - 1 5}$ & 0.46 & 2.40 & & & \\
\cline { 2 - 4 } C-P30 & $\mathbf{6 3 - 1 5}$ & 0.35 & 1.45 & 373 & $3.9 \times 10^{-6}$ & $1.05 \times 10^{-2}$ \\
\hline
\end{tabular}




\begin{tabular}{|c|c|c|c|c|c|c|}
\hline & $30-15$ & 0.17 & 0.47 & & & \\
\hline \multirow{3}{*}{ C-P40 } & $70-10$ & 0.50 & 1.61 & \multirow{3}{*}{253} & \multirow{3}{*}{$5.2 \times 10^{-6}$} & \multirow{3}{*}{$5.20 \times 10^{-3}$} \\
\hline & $50-10$ & 0.36 & 1.10 & & & \\
\hline & $30-10$ & 0.22 & 0.26 & & & \\
\hline
\end{tabular}

As expected from the correlation between static (inhomogeneous) disorder and nanoring size, ${ }^{18}$ there is a trend in the diffusion coefficient values across different nanorings. The best fit for the largest, $c$-P40 nanoring, yields $D=5.20 \times 10^{-3} \mathrm{~cm}^{2} / \mathrm{s}$. This value is increased to $D=1.05 \times 10^{-2} \mathrm{~cm}^{2} / \mathrm{s}$ for $\mathbf{c}-\mathbf{P 3 0}$ and to $D=3.00 \times 10^{-2} \mathrm{~cm}^{2} / \mathrm{s}$ for $\boldsymbol{c}-\mathbf{P 2 0}$. Such values match well with previously reported exciton diffusion coefficients on linear chains of conjugated chromophores. ${ }^{40}$ Bigger nanorings are more disordered and can adopt a broader range of conformations. ${ }^{18}$ This static (on a picosecond time scale) disorder reduces the exciton mobility, ${ }^{41}$ which translates to an increase in the time needed for two excitons to come close enough to annhilate. ${ }^{40}$ Furthermore, the disorder can reduce the coherence length of the exciton, causing it to become more localized and thus smaller. Both phenomena will cause a decrease in annihilation events per unit time, making the value of the parameter $D$ smaller when increasing nanoring size.

Using a one-dimensional random walk approach, it is possible to obtain the diffusion time $\Delta T$ from the ring circumference $L$ and the exciton diffusion coefficient $D$, as shown in Equation 8:

$$
\Delta T=L^{2} / 2 D
$$

$\Delta T$ is the time that it takes for an exciton to perform a full rotation around the ring, here calculated as 113 ps for C-P20, 724 ps for C-P30 and 2.6 ns for c-P40. This is in disagreement with our 35 ps DADS component assignment (see Figure 4) to exciton motion around the nanoring. However, the random walk approach models the diffusion behavior of a point-like particle, while excitons have a finite size that can be as big as ca. 24 porphyrin units. Thus, if we instead use Equation 8 to estimate how long it will take for the center of mass of an exciton to move by one porphyrin site (using $r=1.3 \mathrm{~nm}$ ), we obtain 0.28 ps for $\boldsymbol{c}$-P20, 0.81 ps for $\boldsymbol{c}$ P30 and 1.63 ps for $\mathbf{c - P 4 0 . ~ I t ~ i s ~ r e a s o n a b l e ~ t o ~ a s s u m e ~ t h a t ~ i n ~ t h e ~} 10-50$ ps time window an exciton which was initially generated in an arbitrary position of the ring will be located on a different portion of it, experiencing a different local environment and displaying different photophysics. Thus, the 35 ps decay component present in the global fits can indeed be assigned to exciton motion around the nanoring. 
An alternative approach to the EEA problem, developed by Trinkunas et al..$^{42}$ specifically for ring-shaped structures, was also applied to model our data. In that model, the lifetimes of the transient anisotropy (measured at low pump fluence, see experimental) and of the EEA (measured at high pump fluence) decays are used to estimate the coherence length of the exciton and its hopping time constant; both quantities can, in turn, be related to the diffusion coefficient $D$. This model, applied to our data, did not produce meaningful results (yielding complex values for the exciton size). This could be due to the fact that, in Trinkunas et al., the excited state population decays monoexponentially, while the decay dynamics observed in cP10-40 are non-single exponential. These differences can stem from the fact that, in natural systems, chromophores are held in place by protein scaffolds which keeps static disorder low, while we speculate that the different conformations adopted by the nanorings studied here has an impact on the observed EEA dynamics. Thus, further analysis of the diffusion coefficient recovered above will require detailed simulations of nanoring structural dynamics and their effect on electronic structure.

\section{Conclusions}

Transient absorption on the fs-ns time scale for fully conjugated, alkyne bridged porphyrin macrocycles of 10, 20, 30 and 40 repeat units have been measured as a function of pump fluence, in order to observe and characterize EEA dynamics. The ten-member nanoring ( $c$ P10) does not show any fluence-dependent decay and presents transient absorption spectra that resemble the smallest nanoring of this series $(\boldsymbol{c}-\mathbf{P 6}) .{ }^{34}$ This result is in agreement with the hypothesis of a fully delocalized excitation in c-P6 and c-P10. A change in behavior can be observed when the nanoring size is increased to 20 (or more) porphyrin units. A red-shift is observed in the steady-state absorption spectra and the transient absorption GSB+SE bandwidth narrows for increasing nanoring size, indicating an increase in excitation delocalization. However, this effect saturates and is disrupted due to structural inhomogeneity around the nanoring for bigger structures. Effectively, for bigger nanorings, the coherence size of the exciton becomes independent of ring size. This implies that the exciton is experiencing a local environment which begins to resemble a linear chain, and its coherence length is not bigger than 20 monomer units.

A one dimensional diffusion model developed to describe exciton motion on linear infinite chromophore chains was modified to account for the nanoring size dependent EEA. This model reproduced well the fast decaying component extracted from the experimentally measured intensity dependent data of $\mathbf{c - P 2 0 - 4 0 . ~ T h i s ~ a l l o w e d ~ u s ~ t o ~ e s t i m a t e ~ t h e ~ e x c i t o n ~}$ diffusion coefficients on each nanoring. As expected, the value of $D$ decreases for bigger 
nanorings, due to their more disordered structures which can have a negative impact on both the size and the mobility of the excitons.

\section{Supporting information}

Additional figures including: Evolution Associated Decay Spectra (EADS) of $\boldsymbol{c}-\mathbf{P 1 0} \mathbf{1 0}$, trends of the 1-D exciton diffusion model, rescaled integrated traces at increasing pump fluence for c-P10-40.

\section{Acknowledgments}

This work was supported by EPSRC Grants EP/J009148/1, EP/M016110/1, EP/J007161/1 and J021431/1. MJ thanks Oxford University for a Scatcherd European Scholarship.

\section{References}

(1) Kondo, T.; Jia Chen, W.; S. Schlau-Cohen, G. Single-Molecule Fluorescence Spectroscopy of Photosynthetic Systems. Chem. Rev. 2017, 117 (2), 860-898.

(2) Haver, R.; Tejerina, L.; Jiang, H.-W.; Rickhaus, M.; Jirasek, M.; Grübner, I.; Eggimann, H. J.; Herz, L. M.; Anderson, H. L. Tuning the Circumference of Six-Porphyrin Nanorings. J. Am. Chem. Soc. 2019, 141 (19), 7965-7971.

(3) Donehue, J. E.; Varnavski, O. P.; Cemborski, R.; lyoda, M.; Goodson, T. Probing Coherence in Synthetic Cyclic Light-Harvesting Pigments. J. Am. Chem. Soc. 2011, 133 (13), 4819-4828.

(4) Wan, Y.; Stradomska, A.; Fong, S.; Guo, Z.; Schaller, R. D.; Wiederrecht, G. P.; Knoester, J.; Huang, L. Exciton Level Structure and Dynamics in Tubular Porphyrin Aggregates. J. Phys. Chem. C 2014, 118 (43), 24854-24865.

(5) Didraga, C.; Knoester, J. Chiral Exciton Wave Functions in Cylindrical J Aggregates. J. Chem. Phys. 2004, 121 (2), 946-959.

(6) Parkinson, P.; Kondratuk, D. V.; Menelaou, C.; Gong, J. Q.; Anderson, H. L.; Herz, L. M. Chromophores in Molecular Nanorings: When Is a Ring a Ring? J. Phys. Chem. Lett. 2014, 5 (24), 4356-4361.

(7) Sprafke, J. K.; Kondratuk, D. V.; Wykes, M.; Thompson, A. L.; Hoffmann, M.; Drevinskas, R.; Chen, W.-H.; Yong, C. K.; Kärnbratt, J.; Bullock, J. E.; et al. Belt-Shaped $\pi$-Systems: Relating Geometry to Electronic Structure in a Six-Porphyrin Nanoring. J. Am. Chem. Soc. 2011, 133 (43), 17262-17273.

(8) Adamska, L.; Nayyar, I.; Chen, H.; Swan, A. K.; Oldani, N.; Fernandez-Alberti, S.; Golder, M. R.; Jasti, R.; Doorn, S. K.; Tretiak, S. Self-Trapping of Excitons, Violation of 
Condon Approximation, and Efficient Fluorescence in Conjugated

Cycloparaphenylenes. Nano Lett. 2014, 14 (11), 6539-6546.

(9) Yong, C.-K.; Parkinson, P.; Kondratuk, D. V.; Chen, W.-H.; Stannard, A.; Summerfield, A.; Sprafke, J. K.; O’Sullivan, M. C.; Beton, P. H.; Anderson, H. L.; et al. Ultrafast Delocalization of Excitation in Synthetic Light-Harvesting Nanorings. Chem. Sci. 2015, 6 (1), 181-189.

(10) Gong, J. Q.; Favereau, L.; Anderson, H. L.; Herz, L. M. Breaking the Symmetry in Molecular Nanorings. J. Phys. Chem. Lett. 2016, 7 (2), 332-338.

(11) Cogdell, R. J.; Gall, A.; Köhler, J. The Architecture and Function of the Light-Harvesting Apparatus of Purple Bacteria: From Single Molecules to in Vivo Membranes. Q. Rev. Biophys. 2006, 39 (3), 227-324.

(12) Papillon, D.; Perez, Y.; Caubit, X.; Le Parco, Y. Identification of Chaetognaths as Protostomes Is Supported by the Analysis of Their Mitochondrial Genome. Mol. Biol. Evol. 2004, 21 (11), 2122-2129.

(13) Hildner, R.; Brinks, D.; Nieder, J. B.; Cogdell, R. J.; van Hulst, N. F. Quantum Coherent Energy Transfer over Varying Pathways in Single Light-Harvesting Complexes. Science (80-. ). 2013, 340 (6139), 1448-1451.

(14) Abramavicius, D.; Palmieri, B.; Voronine, D. V.; Šanda, F.; Mukamel, S. Coherent Multidimensional Optical Spectroscopy of Excitons in Molecular Aggregates; Quasiparticle versus Supermolecule Perspectives. Chem. Rev. 2009, 109 (6), 23502408.

(15) Kasha, M. Energy Transfer Mechanisms and the Molecular Exciton Model for Molecular Aggregates. Radiat. Res. 1963, 20 (2), 55-70.

(16) Bolzonello, L.; Fassioli, F.; Collini, E. Correlated Fluctuations and Intraband Dynamics of J-Aggregates Revealed by Combination of 2DES Schemes. J. Phys. Chem. Lett. 2016, 7 (24), 4996-5001.

(17) Halpin, A.; Johnson, P. J. M.; Tempelaar, R.; Murphy, R. S.; Knoester, J.; Jansen, T. L. C.; Miller, R. J. D. Two-Dimensional Spectroscopy of a Molecular Dimer Unveils the Effects of Vibronic Coupling on Exciton Coherences. Nat. Chem. 2014, 6 (3), 196-201.

(18) Kondratuk, D. V.; Perdigaõ, L. M. A.; Esmail, A. M. S.; O'Shea, J. N.; Beton, P. H.; Anderson, H. L. Supramolecular Nesting of Cyclic Polymers. Nat. Chem. 2015, 7 (4), 317-322.

(19) May, V. Kinetic Theory of Exciton-Exciton Annihilation. J. Chem. Phys. 2014, 140 (5), 054103.

(20) Ambrosek, D.; Marciniak, H.; Lochbrunner, S.; Tatchen, J.; Li, X.-Q.; Wurthner, F.; Kuhn, O. Photophysical and Quantum Chemical Study on a J-Aggregate Forming Perylene Bisimide Monomer. Phys. Chem. Chem. Phys. 2011, 13 (39), 17649-17657.

(21) Johnson, A. E.; Kumazaki, S.; Yoshihara, K. Pump-Probe Spectroscopy and Exciton Dynamics of J Aggregates at High Pump Intensities. Chem. Phys. Lett. 1993, 211 (6), 511-515.

(22) Wolter, S.; Aizezers, J.; Fennel, F.; Seidel, M.; Würthner, F.; Kühn, O.; Lochbrunner, S. Size-Dependent Exciton Dynamics in One-Dimensional Perylene Bisimide Aggregates. New J. Phys. 2012, 14 (10), 105027.

(23) Hader, K.; May, V.; Lambert, C.; Engel, V. Identification of Effective Exciton-Exciton Annihilation in Squaraine-Squaraine Copolymers. Phys. Chem. Chem. Phys. 2016, 18 (19), 13368-13374.

(24) Soavi, G.; Dal Conte, S.; Manzoni, C.; Viola, D.; Narita, A.; Hu, Y.; Feng, X.; Hohenester, 
U.; Molinari, E.; Prezzi, D.; et al. Exciton-Exciton Annihilation and Biexciton Stimulated Emission in Graphene Nanoribbons. Nat. Commun. 2016, 7 (1), 11010.

(25) Engel, E.; Leo, K.; Hoffmann, M. Ultrafast Relaxation and Exciton-Exciton Annihilation in PTCDA Thin Films at High Excitation Densities. Chem. Phys. 2006, 325 (1), 170-177.

(26) Ma, F.; Yu, L.-J.; Hendrikx, R.; Wang-Otomo, Z.-Y.; van Grondelle, R. Excitonic and Vibrational Coherence in the Excitation Relaxation Process of Two LH1 Complexes as Revealed by Two-Dimensional Electronic Spectroscopy. J. Phys. Chem. Lett. 2017, 8 (12), 2751-2756.

(27) Megerle, U.; Pugliesi, I.; Schriever, C.; Sailer, C. F.; Riedle, E. Sub-50 Fs Broadband Absorption Spectroscopy with Tunable Excitation: Putting the Analysis of Ultrafast Molecular Dynamics on Solid Ground. Appl. Phys. B Lasers Opt. 2009, 96 (2-3), 215231.

(28) Bols, P. S.; Anderson, H. L. Template-Directed Synthesis of Molecular Nanorings and Cages. Acc. Chem. Res. 2018, 51 (9), 2083-2092.

(29) Kondratuk, D. V.; Perdigao, L. M. A.; O’Sullivan, M. C.; Svatek, S.; Smith, G.; O’Shea, J. N.; Beton, P. H.; Anderson, H. L. Two Vernier-Templated Routes to a 24-Porphyrin Nanoring. Angew. Chemie Int. Ed. 2012, 51 (27), 6696-6699.

(30) Bressan, G.; Green, D.; Chan, Y.; Bulman Page, P. C.; Jones, G. A.; Meech, S. R.; Heisler, I. A. One- to Two-Exciton Transitions in Perylene Bisimide Dimer Revealed by Two-Dimensional Electronic Spectroscopy. J. Phys. Chem. A 2019, 123 (8), 1594-1601.

(31) Knapp, E. W. Lineshapes of Molecular Aggregates, Exchange Narrowing and Intersite Correlation. Chem. Phys. 1984, 85 (1), 73-82.

(32) C. Spano, F. The Spectral Signatures of Frenkel Polarons in $\mathrm{H}$ - and J-Aggregates. Acc. Chem. Res. 2009, 43 (3), 429-439.

(33) Snellenburg, J. J.; Laptenok, S. P.; Seger, R.; Mullen, K. M.; Stokkum, I. H. M. van. Glotaran : A Java -Based Graphical User Interface for the R Package TIMP. J. Stat. Softw. 2012, 49 (3), 1-22.

(34) Bressan, G.; Peeks, M. D.; Anderson, H. L.; Meech, S. R.; Heisler, I. A. Time-Resolved Structural Dynamics of Extended $\pi$-Electron Porphyrin Nanoring. J. Phys. Chem. C 2019, 123 (44), 27222-27229.

(35) Kobayashi, T. J-Aggregates; World Scientific, 2012.

(36) Bressan, G.; Cammidge, A.; Jones, G.; Heisler, I.; González-Lucas, D.; RemiroBuenamañana, S.; Meech, S. Electronic Energy Transfer in a Subphthalocyanine-Zn Porphyrin Dimer Studied by Linear and Nonlinear Ultrafast Spectroscopy. J. Phys. Chem. A 123 (27), 5724-5733.

(37) Peeks, M. D.; Tait, C. E.; Neuhaus, P.; Fischer, G. M.; Hoffmann, M.; Haver, R.; Cnossen, A.; Harmer, J. R.; Timmel, C. R.; Anderson, H. L. Electronic Delocalization in the Radical Cations of Porphyrin Oligomer Molecular Wires. J. Am. Chem. Soc. 2017, 139 (30), 10461-10471.

(38) Zigmantas, D.; Read, E. L.; Mancal, T.; Brixner, T.; Gardiner, A. T.; Cogdell, R. J.; Fleming, G. R. Two-Dimensional Electronic Spectroscopy of the B800-B820 LightHarvesting Complex. Proc. Natl. Acad. Sci. U. S. A. 2006, 103 (34), 12672-12677.

(39) Zamzam, N.; Kaucikas, M.; Nürnberg, D. J.; Rutherford, A. W.; Van Thor, J. J. Femtosecond Infrared Spectroscopy of Chlorophyll F-Containing Photosystem I. Phys. Chem. Chem. Phys. 2019, 21 (3), 1224-1234.

(40) Marciniak, H.; Li, X. Q.; Würthner, F.; Lochbrunner, S. One-Dimensional Exciton Diffusion in Perylene Bisimide Aggregates. J. Phys. Chem. A 2011, 115 (5), 648-654. 
(41) Athanasopoulos, S.; Emelianova, E. V.; Walker, A. B.; Beljonne, D. Exciton Diffusion in Energetically Disordered Organic Materials. Phys. Rev. B 2009, 80 (19), 195209.

(42) Trinkunas, G.; Herek, J. L.; Polívka, T.; Sundström, V.; Pullerits, T. Exciton Delocalization Probed by Excitation Annihilation in the Light-Harvesting Antenna LH2. Phys. Rev. Lett. 2001, 86 (18), 4167-4170. 